\title{
„Make Death Proud to Take Us“: \\ Der Tod der Kleopatra in Literatur und Kunst der Frühen Neuzeit
}

\author{
Achim Aurnbammer
}

\section{Die historische Kleopatra VII.}

Kleopatra VII. (69-30 v. Chr.) ist die letzte ptolemäische Königin in Ägypten. Nachdem Gaius Julius Cäsar sie im Jahre 48 v. Chr. als Königin anerkannt hatte, begleitete sie ihn mit dem gemeinsamen Sohn Cäsarion nach Rom. Nach Cäsars Ermordung kehrte sie nach Ägypten zurück, wo sie bald ein Liebes- und Machtbündnis mit Marcus Antonius schloss, der sie 34 v. Chr. zur „Königin der Könige" ernannte und ihr zuliebe seine römische Ehefrau Octavia, die Schwester Octavians, aufgab. Als Antonius die Seeschlacht bei Actium gegen Octavian, den nachmaligen Kaiser Augustus, verloren und sich selbst getötet hatte, suchte Kleopatra vergeblich, auch Octavian zu becircen. Um nicht in Octavians Triumphzug in Rom als Gefangene präsentiert zu werden, nahm sie sich das Leben. Den Cäsar-Sohn Cäsarion ließ Augustus töten und machte aus dem Ptolemäerreich eine römische Provinz. Bei seinem Triumphzug in Rom führte Augustus ein Bild der Königin mit sich, das sie mit einer Schlange zeigte. ${ }^{1}$

\section{Kleopatras Ende in der römischen Dichtung und Historiographie}

Obwohl kaum authentische Selbstzeugnisse vorliegen, wurde Kleopatra schon früh zu einer Figur, die polarisierte und die Künste zu vielfältiger Deutung zwischen Dämonisierung und Heroisierung anregte. Kleopatras Nachruhm geht vor allem auf Plutarchs Parallelbiographien zurück, wo die Königin knapp in der Cäsar-Vita ${ }^{2}$ und umfassend in der Antonius-Vita ${ }^{3}$ gewürdigt wird. In der AntoniusVita wird das Ende Kleopatras ausführlich geschildert: Zunächst steht sie treu ihrem sterbenden Gemahl Antonius bei und will sich nach dessen Ableben mit einem Dolch gleich selbst umbringen, was ein Römer gerade noch verhindert. ${ }^{4}$ Mit Genehmigung Octavians veranstaltet Kleopatra dann ein öffentliches Totenopfer

1 Vgl. einführend etwa Sabine Kubisch / Hilmar Klinkott (Hg.): Kleopatra. Pharaonin - Göttin - Visionärin, Stuttgart 2011, oder Adrian Goldsworthy: Antony and Cleopatra, London 2010.

2 Plut. Caesar. Die Abkürzungen antiker Autoren und ihrer Werke folgen den Vorgaben in Hubert Cancik u. a. (Hg.): Der Neue Pauly, Bd. 1, Stuttgart 1996, S. XXXIX-XLVII.

3 Plut. Antonius.

4 Ebd., 77, 1-79, 1. 
für Antonius, bei dem sie den Toten anruft, sie wolle mit ihm gemeinsam begraben werden. ${ }^{5}$ Einem Totenmahl folgt schließlich der inszenierte Freitod mit Hilfe von Giftschlangen, die sie in einem Feigenkorb versteckt in ihre Schutzhaft bringen lässt. ${ }^{6}$ Die Sterbeszene beschreibt Plutarch wie ein lebendes Bild: Man findet

Kleopatra schon tot in königlichem Schmuck auf einem goldenen Bett liegen. Die eine von den Frauen, Eiras, verschied eben zu ihren Füßen, die andere, Charmion, die wankte und taumelte, rückte das Diadem, womit das Haupt Kleopatras umwunden war, zurecht. Als einer [von Octavians Boten] im Zorn zu ihr sagte: „Das ist schön, Charmion!“, versetzte sie: „Freilich das Allerschönste, wie es sich für die Nachfahrin so vieler Könige schickt." Weiter konnte sie nichts sagen, sondern sank sogleich neben dem Bett nieder. ${ }^{7}$

Sowohl die szenische Beschreibung als auch die Reaktion Octavians, der „beim Triumph [...] ein Bildnis der Kleopatra mit einer an ihrem Arm hängenden Natter mitführen"8 ließ, vermitteln dem Leser Respekt vor der kühnen Tat:

Ungeachtet nun Caesar [Octavian] über den Tod der Frau sehr ungehalten war, konnte er doch nicht umhin, ihren hohen Sinn zu bewundern und ließ ihren Leichnam neben dem des Antonius mit königlicher Pracht beerdigen. Auch erhielten die beiden Kammerfrauen auf seinen Befehl ein ehrenvolles Begräbnis. ${ }^{9}$

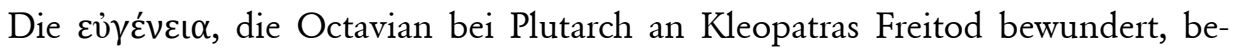
deutet wörtlich ,hohe Abkunft', hier bezeichnet sie die ,edle Tat'. Kleopatras heroischer Tod verbürgt in den Augen ihres politisch-militärischen Gegners Octavian somit ihr Königtum, auch wenn die Dynastie der Ptolemäer mit ihr endet.

\section{Kleopatra in der Frühen Neuzeit}

An Plutarch knüpfte die frühneuzeitliche Kleopatra-Rezeption an. Vom 16. bis zum 18. Jahrhundert war Kleopatra ein beliebtes Bildthema, auch die höfische Oper und das Theater gestalteten ihr Sterben - ein weiblicher Barockheroismus, der im Folgenden exemplarisch konturiert wird.

5 Ebd., 84, 1-4.

6 Ebd., 85, 1-3.

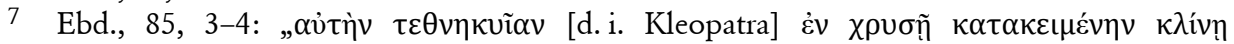

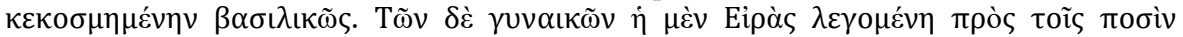

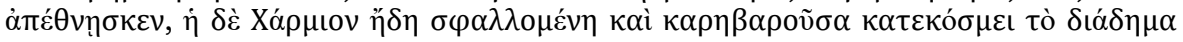

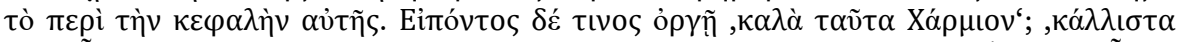

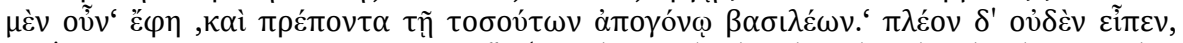

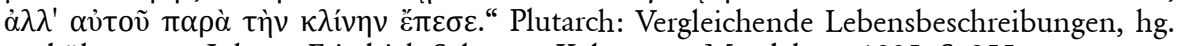
und übers. von Johann Friedrich Salomon Kaltwasser, Magdeburg 1805, S. 255.

Plut. Antonius 86, 1.

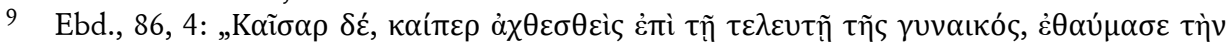

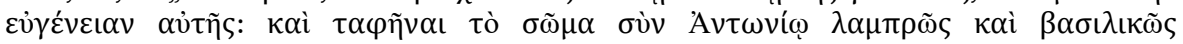

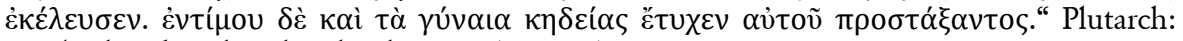
Vergleichende Lebensbeschreibungen (Anm. 7), S. 256. 


\section{Kleopatra in frühneuzeitlichen Viten}

Boccaccio widmet eine Biographie seiner Viten-Sammlung bedeutender Frauen (De claris mulieribus, nach 1351) Kleopatra. Auch wenn er sie negativ beurteilt als „erenata malitiis mulier“ [,eine von Natur aus böse Frau'], wertet er ihre Gestalt insofern auf, als er sie als Einzelperson, unabhängig von Cäsar oder Antonius, würdigt. Bereits hier entscheidet der Tod darüber, ob Kleopatra als Heroine gewertet werden kann oder nicht. Denn Boccaccio gibt zwei Versionen ihres Sterbens wieder. Neben der orthodoxen Version überliefert er als Alternativvariante, Kleopatra sei schon vor der Schlacht bei Actium (31 v. Chr.) gestorben. Antonius habe aus Misstrauen gegenüber Kleopatra nur noch gegessen und getrunken, was vorgekostet worden war. Als Kleopatra ihm bei einem Fest einen vergifteten Becher zum Trinken reichte, habe der argwöhnische Antonius Kleopatra gezwungen, den tödlichen Gifttrank zu leeren. Auch wenn diese Version in der Überlieferung kaum aufgegriffen wurde, erscheint Kleopatra in dieser Sterbelegende als berechnendes Machtweib, das Opfer seiner eigenen Intrige wird. Dieser Wertungsgegensatz, Heldin oder Hexe, bestimmt ihre Rezeption. Dass die Wertungen selbst in ein und demselben Werk uneinheitlich ausfallen können, erweisen die historiographischen Kompilatoren des 16. und 17. Jahrhunderts. Während Cleopatra in der Viten-Sammlung des Johannes Ravisius (1521) als „pessima foemina" 10 bezeichnet wird, zählt sie in Geoffrey Chaucers Legend of Good Women zu einer der heroischen Frauen, deren Würdigung Alkestis in einer Traumvision von dem Dichter einfordert:

And she her death received, with good cheer,

For love of Antony, to her so dear -

And this is history, it is no fable.

Now, where to find a man as reliable,

Who will for love his death so freely take,

I pray God may never our heads so ache!

Amen.

Here ends the legend of Cleopatra, martyr. ${ }^{11}$

Während in England durch Chaucers Vermittlung Kleopatra als Heroine ins kollektive Gedächtnis gerufen wurde, blieb die deutsche Wertungsgeschichte moralisch reservierter. Da Marcus Antonius mit Octavia, der sittsamen Schwester Octavians, verheiratet war, wurde Kleopatra als Ehebrecherin beschuldigt. So schwankt Leonard Mejer in seiner Charakterisierung: „Cleopatra ein schön / geizig und neidig Weib / Königin in Egypten / hatte disen Antonium mit ihrer liebe

10 Johannes Ravisius (Hg.): De memorabilibus et claris mulieribus. Aliquot diversorum scriptorum opera, Paris 1521, F. $50^{\mathrm{r}}-51^{\mathrm{r}}$, hier F. $51^{\mathrm{r}}$. Die Cleopatra-Vita stammt von dem Augustinereremiten Jacobus Philippus Bergomensis.

11 Geoffrey Chaucer: The Legend of Good Women (Poetry in Translation), hg. von Anthony S. Kline, 2008, S. 20-23, hier S. 23. https://www.poetryintranslation.com/PITBR/English/ GoodWomen.php\#anchor_Toc186791812, 21. November 2011, 28. November 2018. 
ganz auff der seiten / daß er thate was sie wolte / er zeügete auch Kinder bej ihren ohngeacht seines Ehweibs Octaviam." 12 Andererseits wird ihre Trauer um ihren Gefährten Antonius zu einem Muster ehelicher Treue über den Tod hinaus überhöht. Wohl nur wenige antike Gestalten wurden so ambivalent beurteilt wie die letzte ptolemäische Herrscherin. Aus christlicher Sicht verurteilte man zwar ihren Selbstmord, relativierte jedoch dessen Diskriminierung durch mildernde Umstände und Erklärungen. So mutmaßt Mejer, weil Augustus ihre erotischen Avancen nicht erwidert habe, „wurd sie kleinmütig und auß verzweiflung ertödet sie sich selbsten mit gifft. Andere meinen durch eine vergiffte Schlangen."13 Nicht nur die Gestalt der Kleopatra, auch ihr Tod wurde Thema einer Kontroverse.

\section{Kleopatra in der Heroik-Debatte um die femmes fortes}

Umstritten war und blieb, inwieweit Kleopatras Tod eine aus christlicher Sicht ruchlose Verzweiflungstat war oder der heroische Akt einer femme forte. In frühneuzeitlichen Bilderserien ,Starker Frauen` erscheint Kleopatra häufig neben Judith und Sophonisbe. ${ }^{14}$

Auch in Madeleine de Scudérys Femmes Illustres (1642) wird die ägyptische Königin als eine bedeutender „Heroines“ porträtiert. ${ }^{15}$ Eine deutsche Übersetzung wurde 1654 von Paris dem Werder vorgelegt, der das Bildnis der Kleopatra mit dem - bereits dem Ausgangstext beigefügten - Motto unterschrieb:

Cette Reine en son mauuais sort,

Comme de la pitié, peut donner de l'enuie;

Puis que la gloire de sa mort,

Oste la honte de sa vie. ${ }^{16}$
O Königinn / bey deiner Noht

Kanstu zugleich / uns Leid und Neid / auf einmal geben:

Dann jener Ruhm / ümb deinen Tod / Benimmt die Schande deinem Leben. ${ }^{17}$

Kleopatra wird hier, aller moralischer Fehltritte zum Trotz, als unnachgiebig Liebende dargestellt, der durch Standhaftigkeit und Stolz heroischer Nachruhm sicher ist.

12 Leonhard Mejer: Theatrum historicum. Oder Historische und Gründliche Erzählung der fürnemsten und nuzlichsten Historien und Geschichten welche sich in der Kirchen Gottes [...] zugetragen, Schaffhausen 1655, S. 126.

13 Ebd., S. 128.

14 Vgl. Patrick Farsen: Kleopatra in der Kunst. Das Bild einer Königin zwischen Geschichte und Mythos, München 2013, S. 95-97: Verweis auf Kleopatras Sterbebild unter den zehn Femmes célèbres im Kardinalspalast der Anne d'Autriche oder im Hôtel de Louis Phélypeaux de Vrillière.

15 [Madeleine de Scudéry]: Les femmes Illvstres [...]. Paris 1642, F. A3 ${ }^{\mathrm{r}}$.

16 Ebd., S. 47.

17 [Madeleine de Scudery]: Viertzig Durchläuchtige Frauen [...], übers. von Paris von dem Werder, Naumburg 1654, F. Eer. 
Dagegen sprach der Jesuit Pierre Le Moyne in seiner Gallerie des Femmes Fortes (1647) Kleopatra den Status einer Heroine ab. Le Moyne polemisierte gegen die libertinen Heroinen eines Torquato Tasso und insistierte auf der chasteté, der Keuschheit, als unabdingbarer weiblicher Tugend. Die Probe aufs Exempel lieferte ihm Kleopatra: Sie habe zwar über Geist und Großzügigkeit verfügt, sei geschickt und zügellos, großmütig und verschwenderisch gewesen. Es sei sehr schade, wenn so große Tugenden so übel beheimatet und so schlimm kombiniert wären:

ie ne voy pas pourquoy l'impudicité des Dames sera iustifiée [...] par l'esprit et par la generosité de Cleopatre: habile et licencieuse, magnanime et débauchée. Il est certes grand dommage, que tant de Vertus ayent esté si mal logées, et en si mauuaise compagnie. ${ }^{18}$

Doch fand die erotische Leidenschaft sowohl in Form der ,beständigen Liebe' als auch als exklusiv aristokratisches Privileg im 17. Jahrhundert ebenso Fürsprecher wie der weibliche Suizid, der als heroische Leistung galt, ebenbürtig dem Freitod eines Seneca oder Sokrates. ${ }^{19}$ Die Aufwertung von Affekten im 17. Jahrhundert verinnerlichte nicht nur das Heroische vom Militärisch-Politischen zu einer affektbasierten Haltung, sondern relativierte auch seine exklusiv männliche Bindung. Wichtiger Gewährsmann für diese Umwertung war Platon, dessen Schriften seit dem Florentiner Neuplatonismus die frühneuzeitliche Liebesphilosophie prägten. So rühmt Phaidros in Platons Symposion Alkestis, die für den geliebten Ehemann Admetos den eigenen Tod auf sich nimmt, als Beispiel einer heroischen Liebe und Inkarnation des Heroischen. ${ }^{20}$

Vor dem Hintergrund dieser Diskussion bietet sich die Gestaltungsgeschichte von Kleopatras Tod als Fallstudie an, um (1) die Motivierung des Freitods, (2) die Heroisierung oder Deheroisierung ihres Sterbens, (3) die Ästhetisierung ihres Sterbens und (4) die jeweilige Bedeutung des Todes für die Bewertung der Gestalt zu untersuchen. Dazu werde ich kurz auf die Kleopatra-Ikonographie des

18 Pierre Le Moyne: La Gallerie des Femmes Fortes, Paris 1647, S. 174. Vgl. ebd.: La Femme Forte, Ode premiere, F. ee $4^{\mathrm{r}}$-ee $4^{\mathrm{v}}$ : „Il luy souuient de Cleopatre, / Dont le celebre desespoir, / Encor auiourd'huy se fait voir, / Auec pompe sur le Theatre. / Elle mit à prix la Beauté; / Prostitua la Royautè; / Abusa des thresors de la terre et de l'onde: /Et par vn luxe enorme et fatal à sa Cour, / Ses Ayeux auoient fait les Miracles du Monde, / A beaucoup moins de frais qu'elle ne fit l'amour." Siehe ebd., S. 12: Vergleich mit Tiberius und Caligula; S.72: Vergleich mit Mariamne; S. 150-152: Zenobia als Nachkommin der Cleopatre; S. 174: Kritik an Tassos Heroinen und Forderung der chasteté als Voraussetzung der Heroisierung; S. 357: Marie Stuart als moderner Gegentypus.

19 Vgl. Philippe Bousquet: Le suicide féminin au XVII e siècle. Un acte héroique?, in: Richard G. Hodgson (Hg.): La femme au XVII ${ }^{\mathrm{e}}$ siècle. Actes du colloque de Vancouver, Tübingen 2002, S. 183-200. Zur „beständigen Liebe als heroischer Selbstrücknahme“ vgl. Martin Disselkamp: Barockheroismus. Konzeptionen ,politischer ${ }^{\circ}$ Größe in Literatur und Traktatistik des 17. Jahrhunderts, Tübingen 2002, S.348. Zur Heroisierung des Freitods von Seneca vgl. den Beitrag von Peter Eich, Sitta von Reden und Anna Schreurs-Morét in diesem Band.

20 Plat. symp. 179b; vgl. Achim Geisenhanslücke: Die Sprache der Liebe. Figurationen der Übertragung von Platon zu Lacan, Paderborn 2016, S. 31. 
17. Jahrhunderts eingehen, um dann exemplarisch die Sterbeszenen in drei Kleopatra-Dramen des 17. Jahrhunderts zu untersuchen.

\section{Kleopatra-Ikonographie}

Die Kleopatra-Ikonographie in Renaissance und Barock dominieren zwei Episoden: Ihre Verschwendungssucht in Gestalt der bei Plinius überlieferten sogenannten Sesterzenwette, einem exotischen Festmahl, bei dem Kleopatra eine in Essig aufgelöste Perle trinkt, ${ }^{21}$ und - weitaus häufiger - die Freitod-Darstellungen, die in zwei unterschiedlichen Typen vorkommen: Zum einen als repräsentative Darstellung, welche die sterbende Kleopatra in königlichem Ornat in einer höfischen Öffentlichkeit zeigt, zum andern als intime Szene des Freitods, die sich auf die sterbende Königin mit ihren beiden Dienerinnen beschränkt oder diese sogar weglässt. ${ }^{22}$

Ungewöhnlich ist das Gruppenbild von Alessandro Turchi (um 1640; Abb. 1). Es inszeniert den doppelten Selbstmord Marcus Antonius' und Kleopatras als gemeinsamen Liebestod: Die beiden bleichen Körper bestimmen die Komposition. Alessandro Turchi präsentiert den entseelten Leichnam Marcus Antonius' auf einer Bank im Vordergrund, am Boden liegt der Dolch, mit dem er sich getötet hat. Eine jugendliche Repoussoir-Figur links verhüllt den Toten, während ihn ein betender Mann im Hintergrund betrauert. Die sterbende Kleopatra dominiert mit fünf Assistenzfiguren die Szene. Die Verbindung zu dem toten Antonius stiftet der gesenkte Blick der schon geschwächten Kleopatra, die ihre Brust entblößt hat und in der Rechten die tödliche Giftschlange hält. Ihren Leib stützen die zwei bei Plutarch überlieferten Dienerinnen. Eine erschrocken-bewundernde und eine weinende Frau am rechten Bildrand geben dem Betrachter das empathisch-admirative Rezeptionsmuster vor. Die Inszenierung des Freitods als ein heroischer Liebesund Treuebeweis, wie sie Turchi für eine Serie von „außergewöhnlichen Gestalten der Antike“23 im Hôtel de Louis Phélypeaux de la Vrillière malte, blieb allerdings eine ikonographische Ausnahme.

Die meisten frühneuzeitlichen Darstellungen heben auf Kleopatras weibliche Schönheit ab und stilisieren ihr Sterben im weiblichen Gruppenbild zu einer rückhaltlosen Hingabe an den Tod. Ein Beispiel dafür ist Guido Cagnaccis Gemälde (Abb. 2): Es zeigt die sterbende Kleopatra auf einem rot bespannten Thron, umgeben von sechs Dienerinnen (um 1660). ${ }^{24}$ Die Mischung von Repräsentativität und Intimität zeigt sich im Habitus Kleopatras: Eine Krone ziert ihre zusam-

21 Plin. nat. 9, 119-121.

22 Ein Einzelporträt, wie es von Michelangelo überliefert ist, könnte etwa durch die Überlieferung von Octavians Triumphzug motiviert sein.

23 Farsen: Kleopatra in der Kunst (Anm. 14), S. 98.

24 Ebd., S. 177-178. 


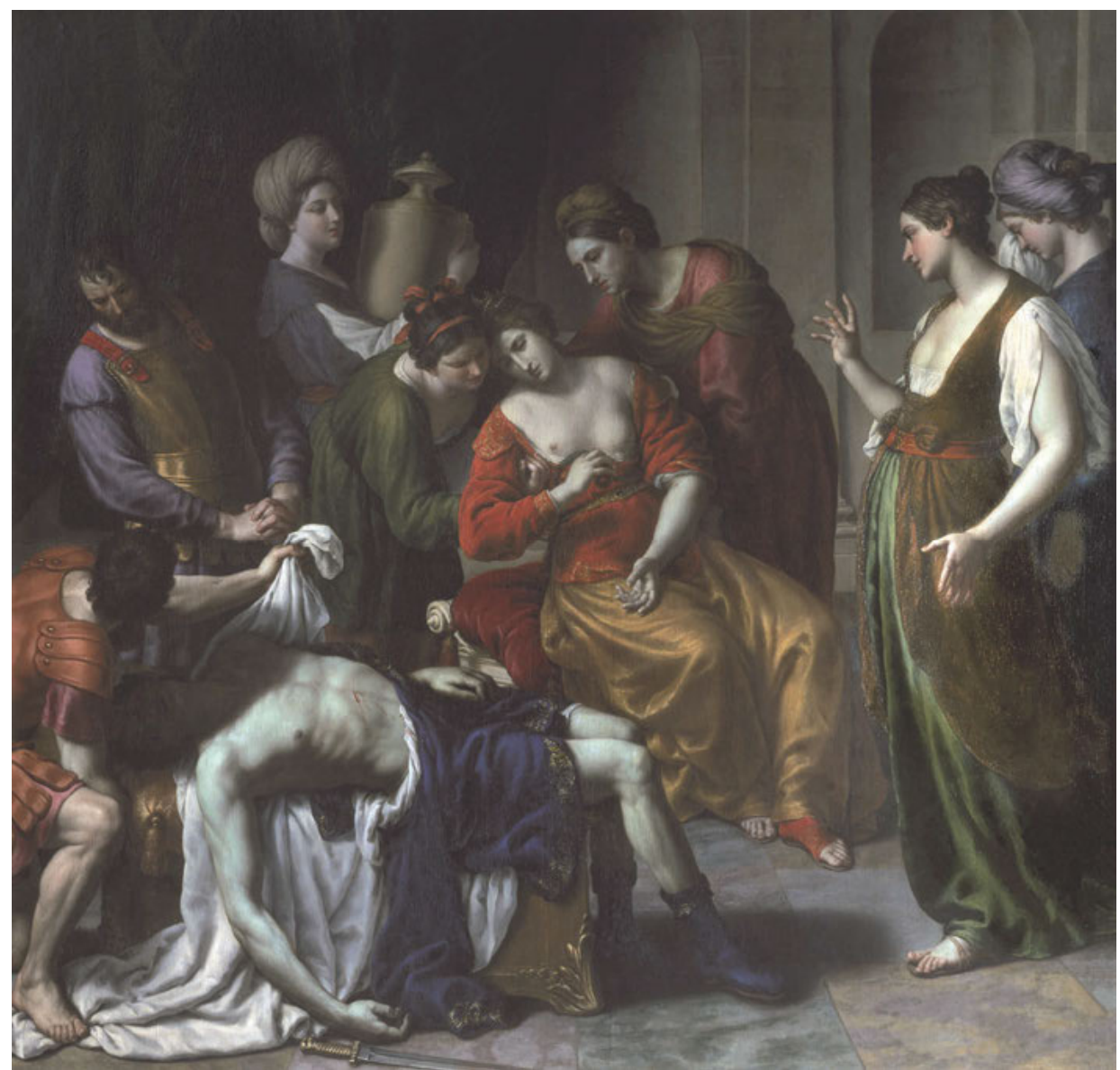

Abb. 1: Alessandro Turchi, La Mort de Cléopâtre, Öl auf Leinwand, um 1640, Paris, Musée du Louvre, Inv. 703.

mengebundenen Haare, während ihr blaues Gewand und weißes Unterhemd bis zur Scham heruntergelassen sind. Eine Schlange, die sich um den rechten Arm ringelt, beißt in die Armbeuge. Die zwei außenstehenden Dienerinnen - möglicherweise Eiras und Charmion, die mit der Königin in den Tod gehen -, haben ihre Oberkörper ebenfalls entblößt, was die Intimität der Szene unterstreicht. Die Dienerinnen repräsentieren unterschiedliche Trauerreaktionen, Pathosformeln, die von Verzweiflung über Erschrecken, Weinen und gefasstes Beten reichen. Sie beglaubigen zugleich im Kontrast mit dem ruhig gefassten Antlitz Kleopatras deren königlich-heroische Natur.

Mit derartigen Intimisierungen ging auch eine Erotisierung der Sterbeszene einher, verdeutlicht durch den sexuell konnotierten Einsatz der Schlange, die in der christlichen Symbolik auf die Paradiesgeschichte verweist. So beißt die Schlange in vielen Darstellungen Kleopatra nicht, wie bei Plutarch überliefert, in 


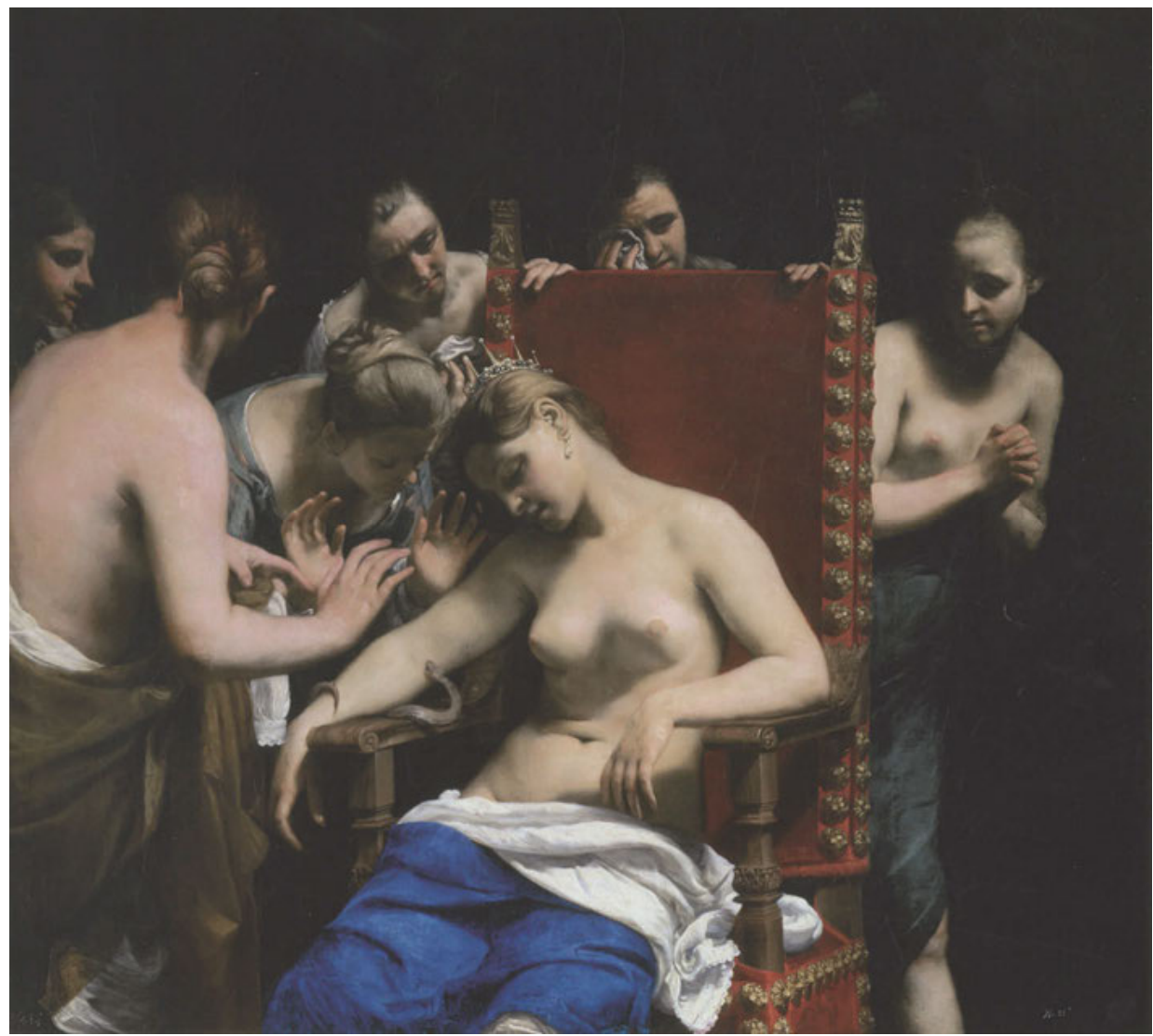

Abb. 2: Guido Cagnacci, Der Tod der Kleopatra, Öl auf Leinwand, um 1659-1662, Wien, Kunsthistorisches Museum, Gemäldesammlung, Inv. 260.

den Arm, sondern wie in Giovanni Francesco Guerrieris Porträt (1630/40) in die entblößte Brust (Abb. 3). Gerade der königliche Habit mit Krone, edlem Gewand, Spitzenunterkleid, Schmuck an Ohren, Händen und Fingern und dem gefassten Gesichtsausdruck schafft einen frappierenden Gegensatz zum halb enthüllten Busen mit der Schlange. Auf diese Weise tendiert die Sterbeszene zunehmend zum weiblichen Akt: Diesen Bildtyp repräsentiert etwa die jugendlich wirkende Kleopatra in Massimo Stanziones Tod der Kleopatra (um 1630; Abb. 4). Hier ist Kleopatra bar jeglicher königlichen Insignien und bekundet ihre Heroik nur in ihrer noblen Haltung: Während sie sich mit der Rechten abstützt, verhüllt sie mit der Linken, in der sie auch eine kleine Schlange hält, im Stile einer Venus pudica die Scham mit einem purpurfarbenen Tuch. Den Kopf nach rechts gewendet, zeigen die geschlossenen Augen Todesbereitschaft an. Die Sterbende gibt sich dem voyeuristischen Betrachter in ihrer Nacktheit preis. Indem sie aber auch im Akt des Sterbens ihre Haltung, Würde und Schönheit bewahrt und angstlos dem Tod begegnet, unterstreicht sie ihre Unerschütterlichkeit und 


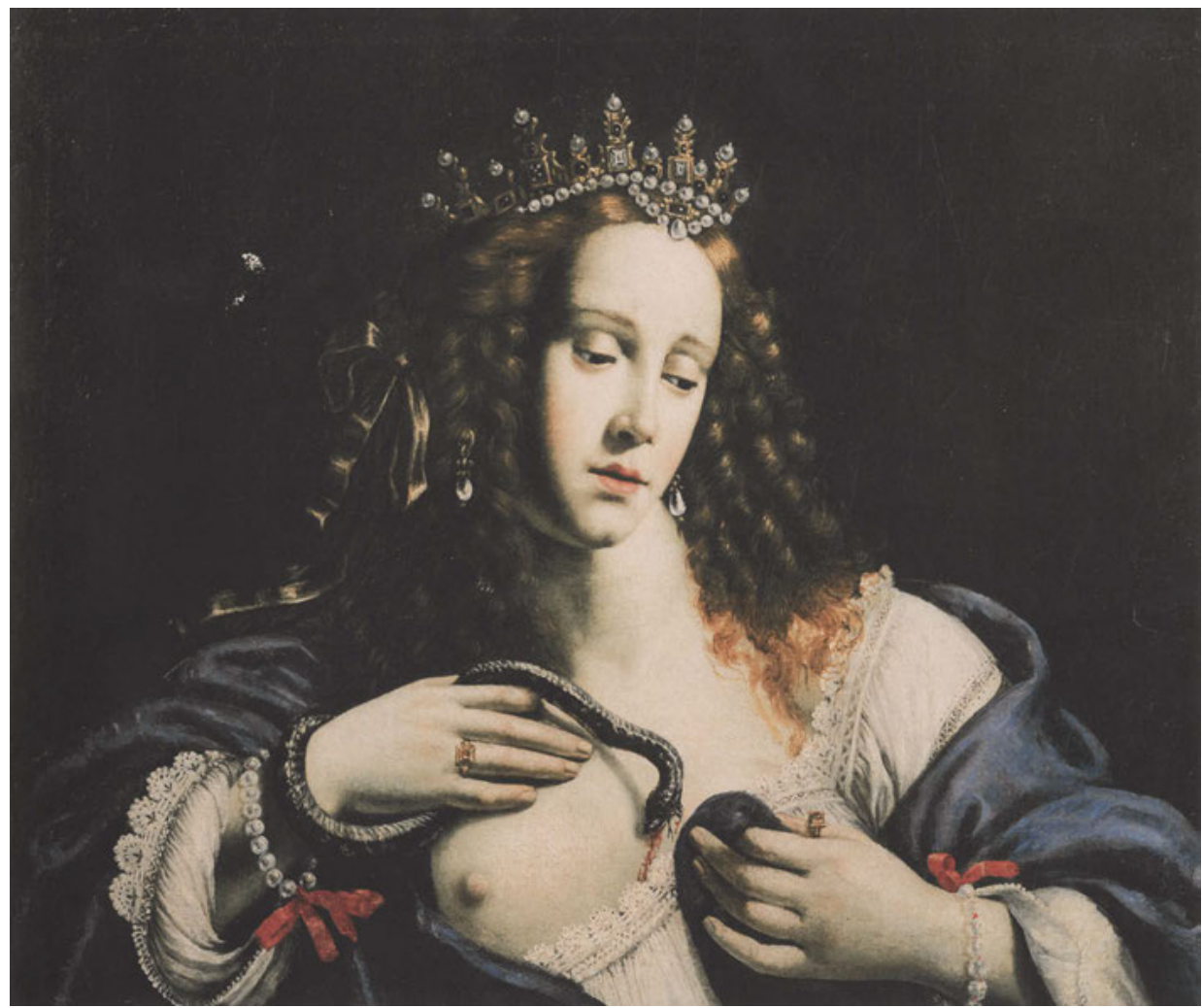

Abb. 3: Giovanni Francesco Guerrieri, Kleopatra, 1600-1630, Öl auf Leinwand, Fano, Quadreria della Fondazione Cassa di Risparmio di Fano, ohne Inv.

repräsentiert einen „Beispielheroismus weiblicher Ehre“. ${ }^{25}$ Guido Reni, der das Sujet mehrfach gestaltet hat, nähert die sterbende Kleopatra - wie in diesem Gemälde (um 1626) - den bildlichen Repräsentationen von Märtyrerinnen an (Abb. 5): Immer ist Kleopatras Blick nach oben gerichtet und die Erwartung des Todes als Jenseitshoffnung gedeutet. Dementsprechend hat Reni auch bis auf die Schlange alle sonstigen Attribute, Schmuck und Assistenzfiguren beiseitegelassen, so dass nur der Moment des Übergangs vom Leben in den Tod dargestellt und im märtyrergleichen Gesichtsausdruck sowie in der in sich ruhenden Haltung als ,heroische Selbstrücknahme` inszeniert wird. ${ }^{26}$

Sämtliche Darstellungstypen, so können wir unseren kleinen kunstgeschichtlichen Überblick zusammenfassen, präsentieren somit Kleopatra als Muster einer weiblichen Heroine, die wie die karthagische Königin Dido, von Aeneas verlassen, ihrem Leben aus Liebe ein Ende setzt und angstlos in den Tod geht. Die

25 Bruno Markwardt: Geschichte der deutschen Poetik, Bd. 1. Barock und Frühaufklärung, Berlin ${ }^{3} 1964$, S. 171.

26 Disselkamp: Barockheroismus (Anm. 19), S. 348. 


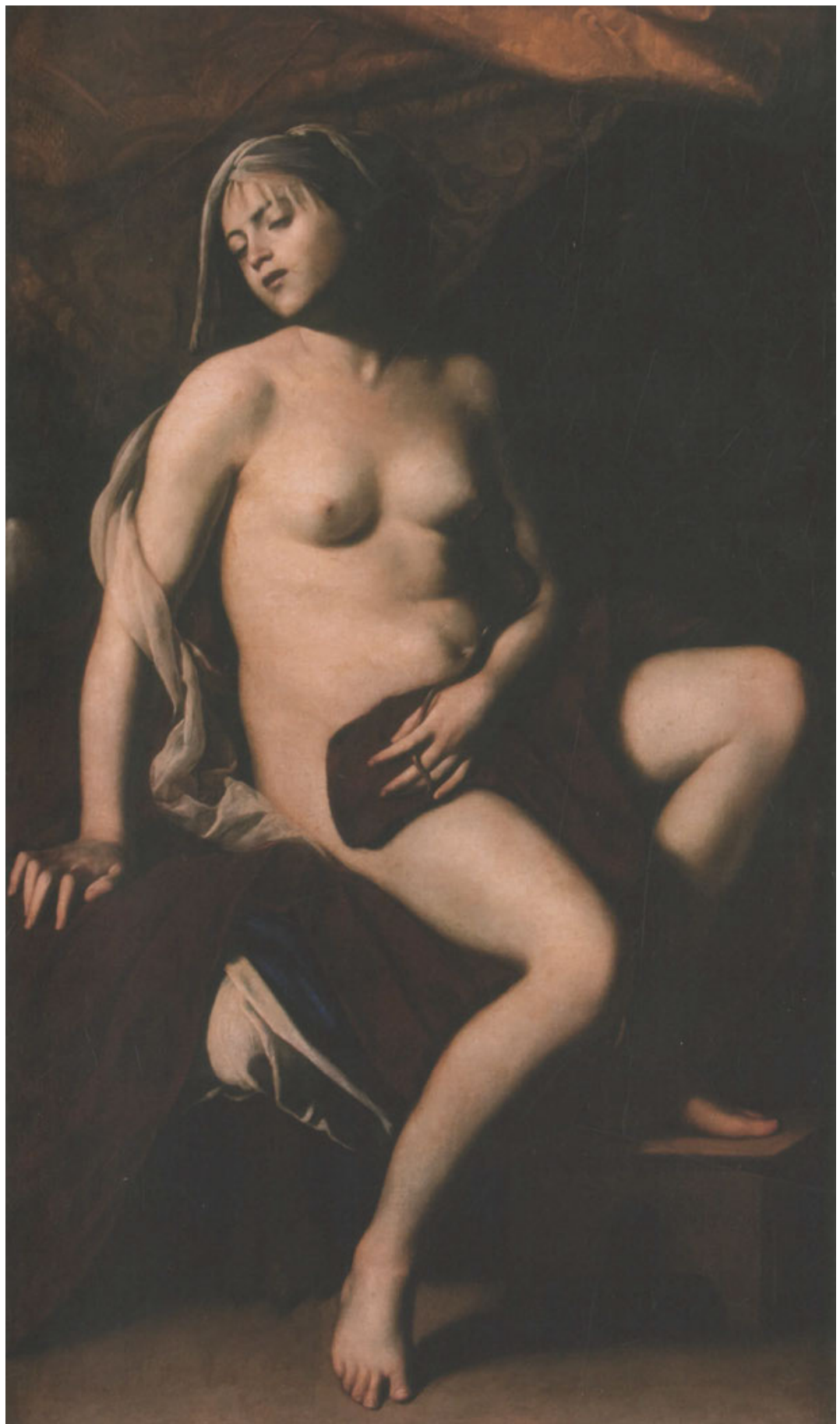

Abb. 4: Massimo Stanzione, Kleopatra, 1630-1640, Öl auf Leinwand, St. Petersburg, Staatliche Eremitage, Inv. ГЭ-10030. 


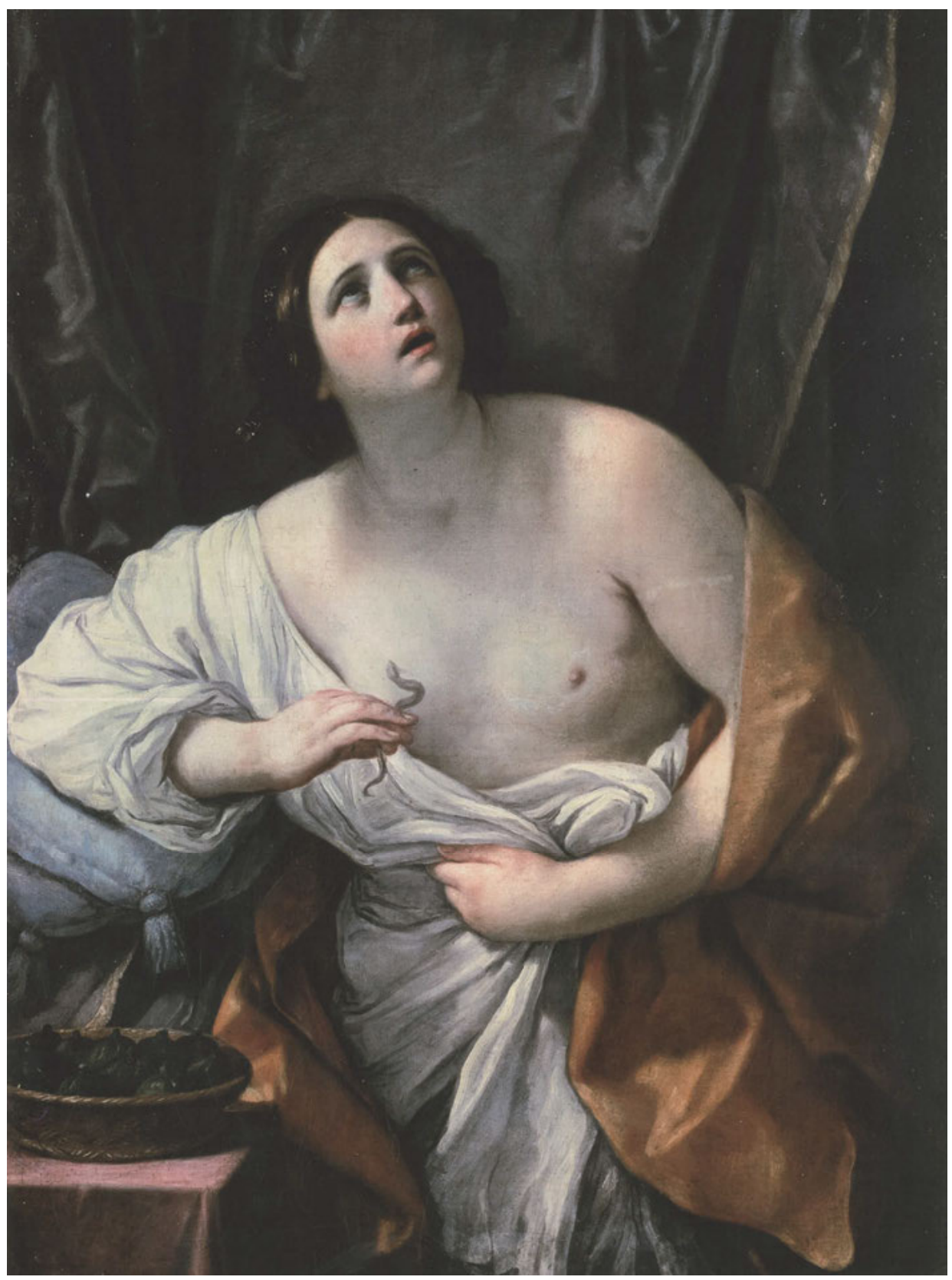

Abb. 5: Guido Reni, Der Tod der Kleopatra, Öl auf Leinwand, um 1630, Florenz, Galleria Palatina di Palazzo Pitti, Inv. 270. 
Aufwertung der Affekte und die Psychologisierung des Heroischen spiegeln sich in einer Ästhetisierung, Erotisierung und Sakralisierung von Kleopatras Freitod.

Inwiefern das Modell einer heroischen Liebe, die sich im unerschrockenen Freitod aus Liebe verbürgt, auch die dramatischen Inszenierungen der KleopatraGestalt prägte, möchte ich anhand dreier Kleopatra-Tragödien des 17. Jahrhunderts genauer prüfen: Das Trauerspiel, neben dem Epos die vornehmste literarische Gattung, ist das maßgebliche literarische Medium der Frühen Neuzeit, das konkurrierende Formen des Heroischen performativ in Szene setzte und dialogisch erprobte.

Die drei Trauerspiele: Antony and Cleopatra ([1608] 1623) von William Shakespeare, Cleopatra (1. Version 1661, 2. Fassung 1680) von Daniel Casper von Lohenstein und All for Love or the World Well Lost (1678) von John Dryden sind Ausdruck einer gesamteuropäischen Geschmacksmode. Zwischen 1600 und 1750 dürften an die hundert Kleopatra-Tragödien und Opern auf europäischen Bühnen gespielt worden sein. ${ }^{27}$ Ich möchte im Folgenden untersuchen, inwieweit der Freitod der ägyptischen Königin in den Tragödien charakterlich oder situativ motiviert ist und ob und wie er jeweils heroisiert wird.

\section{Kleopatra-Tragödien des 17. Jabrbunderts}

Shakespeare: Antony and Cleopatra $(1608[1623])^{28}$

William Shakespeares Tragödie Antony and Cleopatra aus dem Jahre 1608, ${ }^{29} 1623$ erstmals gedruckt, hebt ganz auf die Todesszenen der beiden Protagonisten ab. Der vierte Akt ist Antonys Suizid gewidmet. Antony stürzt sich nach der fingierten Nachricht Cleopatras, sie wäre mit den Worten „Marc Anton“ auf den Lippen gestorben, in sein Schwert. Der Schlussakt konzentriert sich auf das Ende Cleopatras. Sie hat ihren Freitod möglicherweise schon mit der folgenreichen Botschaft an Antony ins Werk gesetzt, spätestens aber mit Antonys Ableben, der sterbend zu ihr gebracht wurde: Nachdem dieser in seinen letzten Worten gewünscht hat, er möge als „the greatest prince o'th' world / The noblest“30 der Nachwelt im Gedächtnis bleiben, nimmt Cleopatra diesen Wunsch auf. So vervollständigt sie Antonys letztes Wort „I can no more“ mit der Anrede „Noblest

27 Die Frage nach der Heroik der Kleopatra-Figur fand in der Rezeptionsforschung bislang wenig Beachtung.

28 Textgrundlage der folgenden Ausführungen ist: William Shakespeare: Antony and Cleopatra (The Arden Edition of The Works of William Shakespeare), hg. von M. R. Ridley, London 1976.

29 Für die Datierung ist neben der Konkurrenz zur Neubearbeitung von Samuel Daniels Cleopatra (zuerst 1594, dann 1607) der Eintrag im Stationer's Register (20. Mai 1608) maßgeblich.

30 Shakespeare: Antony and Cleopatra (Anm. 28), IV, 15, 54-55. 
of men, woo't die“ ${ }^{\text {311 }}$ zu einem Blankvers und kündigt in der Schlussszene des vierten Aktes ihren Dienerinnen verschlüsselt ihren Plan an:

We'll bury him: and then what's brave, what's noble,

Let's do it after the high Roman fashion

And make death proud to take us. [... $]^{32}$

Damit erklärt Cleopatra, den ,edlen' Freitod ihres römischen Mannes zum Präfigurat ihres eigenen Sterbens zu machen. Noble, das Zentralwort in Antonys Sterbeszene, hatte dieser bereits gebraucht, um das Verhalten seines Pagen Eros zu charakterisieren. Dieser hatte sich seinem Befehl widersetzt, als er nicht ihn, sondern sich selbst tötete, und damit Antony zu der imitatio heroica veranlasst, es Eros gleichzutun und sich selbst zu töten:

[...] Thrice-nobler than myself!

Thou teachest me, $\mathrm{O}$ valiant Eros, what

I should, and thou couldst not. My queen and Eros

Have by their brave instruction got upon me

A nobleness in record: $[\ldots]^{33}$

Tatsächlich inszeniert Cleopatra ihren Freitod als königliche Hochzeit: Sie beauftragt ihre Dienerinnen, sie königlich („queen“) einzukleiden wie vor der legendären liebestiftenden Begegnung mit Antonius: „Bring our crown and all“. ${ }^{34}$ Dass Cleopatra dem römischen Sieger Octavian eine Komödie vorspielt und längst zum Freitod entschlossen ist, zeigt ihre Reaktion auf den bäuerischen Clown, der unter dem Vorwand, Feigen zu liefern, die tödlichen Nattern bringt. Entschlossen, eine ,edle Tat zu vollbringen“ („do a noble deed!“), ${ }^{35}$ verkündet sie:

My resolution's placed, and I have nothing

Of woman in me. Now from head to foot

I am marble-constant. Now the fleeting moon

No planet is of mine. $[\ldots]^{36}$

Cleopatra wechselt hier in die Rolle einer entschlossenen femme forte, wie sie sie in den ersten drei Akten der Tragödie verkörperte. Sie entsagt in dieser Situation der weiblichen Liebe und dem Leben, und ihre Selbstcharakterisierung als ,marmorfest“ deutet ihre Monumentalisierung als Grabskulptur neben ihrem Geliebten

\footnotetext{
Ebd., IV, 15, 58-59.

Ebd., IV, 15, 86-88.

Ebd., IV, 14, 95-99.

Ebd., V, 2, 231.

Ebd., V, 2, 236.
}

6 Ebd., V, 2, 237-240. Es ist wohl ein Spezifikum der englischen Frühen Neuzeit, dass Geschlechtsunterschiede in der Heroisierung eine weniger wichtige Rolle spielen als in den anderen europäischen Literaturen. Zwar ist heroisches Handeln auch in England ,männlich' kodiert, doch Frauen steht die Usurpation dieser ,männlichen' Eigenschaften prinzipiell offen. 
Antony voraus. ${ }^{37}$ Der Entschluss zum Freitod, nicht erst dessen Ausführung, erhöht Kleopatra bei Shakespeare zur Heroine. Mit dem sukzessiven Rückzug aus dem Leben in den Tod geht paradoxerweise ein Übergang in die Unsterblichkeit einher: Mit der Investitur zur Königin von Ägypten vollzieht sich eine Wendung zur über den Tod hinaus liebenden Frau, die über das Hören und Sehen des toten Ehemanns zur imaginären körperlichen Begegnung führt:

Give me my robe. Put on my crown. I have

Immortal longings in me. [...]

[...] Methinks I hear

Antony call. I see him rouse himself

To praise my noble act. [...]

$[\ldots]$ - Husband, I come!

Now to that name my courage prove my title! $!^{38}$

Selbst Octavian zollt der Toten Respekt mit den Ausrufen: „Bravest at the last“ (,Ihr End erhaben $)^{69}$ und „o noble weakness“ (,edle Schwäche) ${ }^{40}$ Als besiegter Sieger bekräftigt er die heroische Qualität des Doppelselbstmordes und ordnet ein Staatsbegräbnis an für „a pair so famous“. ${ }^{41}$ Zugleich akzeptiert er, dass das Mitleid für das tragische Schicksal sich mit seinem militärischen Ruhm die Waage hält. ${ }^{42}$ Tatsächlich aber überschreitet die heroische Todesinszenierung der beiden Protagonisten bei Shakespeare, nicht zuletzt auch durch die zu Totenopfern stilisierten Freitode ihrer Getreuen (Eros sowie Eiras und Charmion), als Zeugnis einer unbedingten Liebesleidenschaft das kalte Machtkalkül Octavians. ${ }^{43}$

\section{Daniel Casper von Lohenstein: Cleopatra (1661, rev. Fassung 1680) ${ }^{44}$}

Daniel Casper von Lohenstein hat sein Trauer-Spiel Cleopatra (Abb. 6) unter ein Zitat aus Tacitus' Historien gestellt, in dem der römische Kaiser Vitellius von seinen Vertrauten vor seinem Widersacher Vespasian gewarnt wird:

37 Vgl. John M. Bowers: I am Marble-Constant, in: Huntington Library Quarterly 46, 1983, S. 283-297, hier S. 289. Vgl. außerdem: Anne Barton: Nature's Piece 'gainst Fancy. The Divided Catastrophe of Antony and Cleopatra, London 1973, S. 20. Auch Barton folgert aus diesem Selbstvergleich, Cleopatra inszeniere ihre Suizidszene selbst als lebendes Kunstwerk, um ihren Tod nach ihrem Sinne zu glorifizieren.

Shakespeare: Antony and Cleopatra (Anm. 28), V, 2, 279-287.

Ebd., V, 2, 334.

Ebd., V, 2, 343.

Ebd., V, 2, 358.

42 „CAESAR: High events as these / Strike those that make them, and their story is / No less in pity than his glory which / Brought them to be lamented." Ebd., V 2, 358-361.

43 Vgl. dazu Uwe Baumann: Vorausdeutung und Tod im englischen Römerdrama der Renaissance (1564-1642). „The heavens themselves blaze forth the death of princes“, Tübingen/ Basel 1996, bes. S. 187-194, hier S. 192.

Textgrundlage der folgenden Ausführungen ist: Daniel Casper von Lohenstein: Cleopatra. Erstfassung [1661], in: Sämtliche Werke. Abteilung II, Dramen, Bd. 1,1: Text, hg. von Lothar Mundt, Berlin/New York 2008, S. 141-390. 


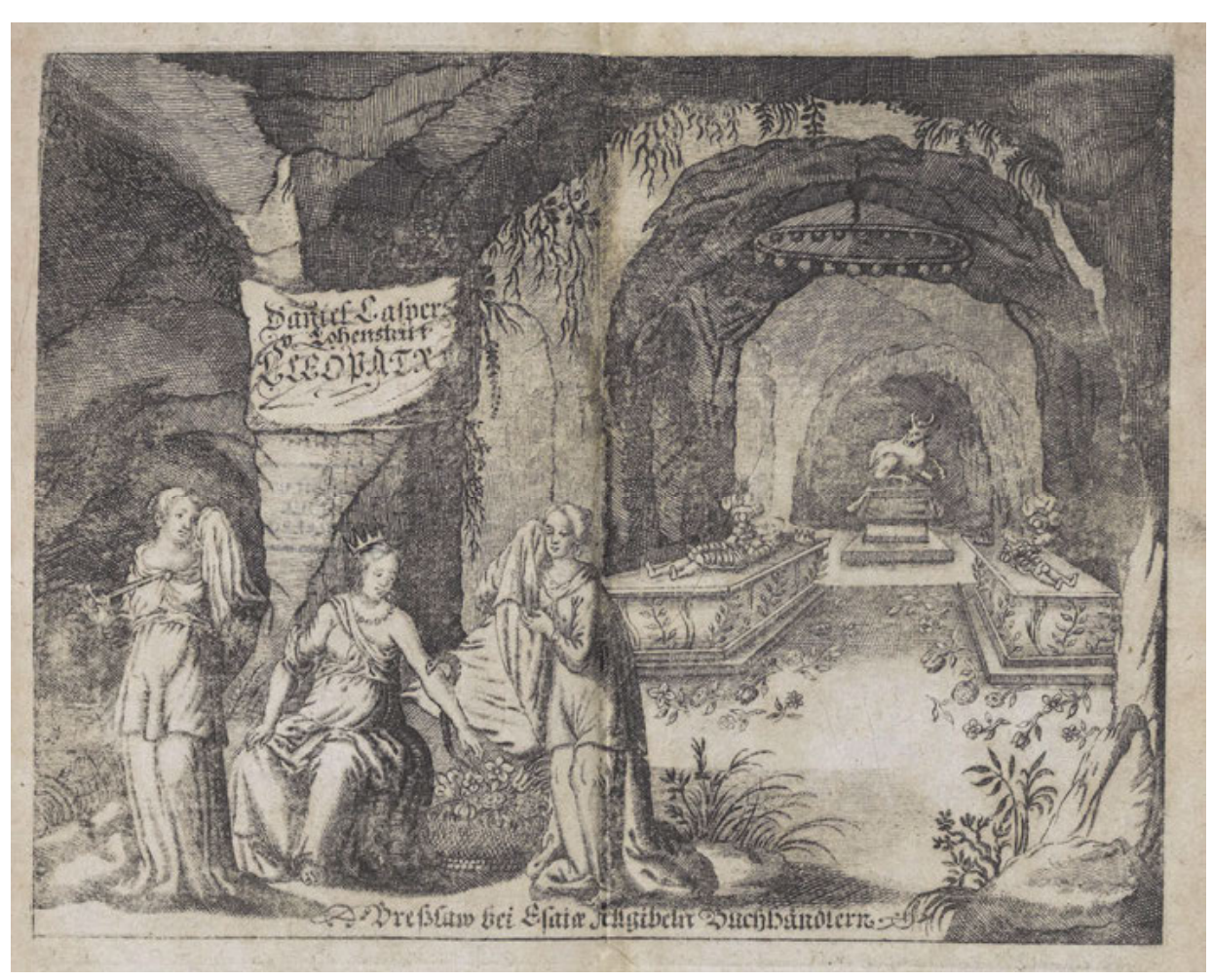

Abb. 6: Daniel Casper von Lohenstein, Cleopatra. Trauer-Spiel, Frankfurt/Leipzig 1724, Frontispiz.

sterben müssen wir, wenn wir besiegt werden, sterben müssen wir, wenn wir uns ergeben. Die einzige Frage ist, ob wir den Geist in Schande und Verachtung aufgeben oder in mutigem Kampf. ${ }^{45}$

Mit dieser fatalistischen Devise, die einzig auf die Haltung in einer aussichtslosen Situation abhebt, einer Situation, in der die Agency eingeschränkt ist, steckt Lohenstein den Deutungsrahmen seiner tragischen Behandlung der CleopatraGestalt ab: Cleopatra wird von Lohenstein als eiskaltes Machtweib und politische Intrigantin inszeniert. Ihren politischen Zielen ordnet sie moralische Werte unter und setzt dafür alles, vor allem ihre erotische Attraktivität ein: So versucht sie nach dem Tod des Antonius, den Lohenstein in den dritten Akt vorzieht, auch noch Octavius (Namensform für Octavian bzw. Augustus) für sich zu gewinnen. Diese Verführungsszene nimmt bei Lohenstein breiten Raum ein:

45 Tac. hist. 3, 66: „moriendum victis, moriendum deditis: id solum referre, novissimum spiritum per ludibrium et contumelias effundant an per virtutem." P. Cornelius Tacitus: Liber Tertivs, in: Historien / Historiae, hg. und übers. von Joseph Borst, Berlin/Boston ${ }^{7} 2010$, S. 342 . 
Ich brenn'! ich brenn'! August! denn durch des Keisers Glider /

Zeugt sich mein Julius mein Julius sich wider.

Di Flamme / di mit ihm schon in der Asche lag /

Bekommet frisches Oel. ${ }^{46}$

Cleopatra spricht hier Octavius als Verwandten und Wiedergänger ihres ersten römischen Mannes, Gaius Julius Cäsar, an, und fordert ihn auf, ihre leiblichen Vorzüge zu erproben. Zuvor hatte allerdings Cleopatra gekonnt und ,lebensecht ${ }^{6}$ ihren eigenen Tod als „Schau-Spiel“47 inszeniert, um Antonius zum Freitod zu bewegen und so wieder politisch frei handeln zu können, wie sie ihre Dienerin Charmium instruiert:

Daß / wenn mein schlaffend Leib wird als entseelet ruh'n /

Anton den falschen Todt als wahrhafft stracks erfahre;

Geh hin! dein und mein Heil wächst auß der Todten-Baare. ${ }^{48}$

Antonius reagiert nach Cleopatras Plan. Auf die Nachricht vom vermeintlichen Ableben Cleopatras entschließt er sich zum Suizid, den er als Liebestod ankündigt, indem er die totgeglaubte Geliebte anspricht und vom apostrophierten Tod eine Vereinigung im Jenseits erbittet:

Cleopatra mein Licht! Cleopatra mein Leben!

Du Seele meiner Seel'! umb deinen Schatten schweben /

Di Lebens-Geister schon / di mich di heisse Noth /

Dir aufzuopffern zwingt / komm' angenehmer Todt

Erwünschter Jammer-Port! ich suche dein Gestade;

Wer deine Küsten kiest / der seegelt recht gerade /

Den Glückes-Inseln zu. Cleopatra mein Licht!

Ach! ich erblicke schon dein sternend Angesicht!

[...]

Komm Schwerdt! Komm süsser Todt! Vermähle mich mit ihr. ${ }^{49}$

Als aber der sterbende Antonius zu Cleopatra in die „Königliche Todten-Grufft“, dem zentralen Schauplatz der Tragödie, gebracht wird, will auch sie ihrem Leben ein Ende setzen und entsagt dem Gift nur, weil Antonius ausdrücklich wünscht, sie möge ihn in der „Grufft der Ptolomeer“ beisetzen. ${ }^{50}$

Die fünfte Abhandlung ist ganz Cleopatras Freitod gewidmet. Cleopatra vollzieht mit ihren Dienerinnen Eiras und Charmium eine Totenfeier für Antonius, den „Ausbund aller Helden“:51 Sie balsamieren den Leichnam und setzen ihm ein ehrendes Epitaph ${ }^{52}$ bevor Cleopatra ihren Freitod als ,zweite Hochzeit' an-

46 Lohenstein: Cleopatra (Anm. 44), IV, 409-412.

47 Ebd., III, 61.

48 Ebd., III, 64-66.

49 Ebd., III, 369-376 und 396.

50 Ebd., III, 559.

51 Ebd., V, 7.

52 „Und grabt sein redend Lob in stumme Marmel ein: / Hier lig't Egiptens Heil / di Freiheit Rom's umbfangen. / Denn beider Wolfahrt ist mit dem Anton vergangen." Ebd., V, 26-28. 
kündigt: „Cleopatra sol itzt noch einmal durch den Tod / Sich dem Anton vermähln“ und „durch ihr Blutt entdecken: / Daß knecht'sche Geister nicht in disen Adern stecken". ${ }^{53}$ Allerdings ist ihr Motiv weniger die Liebe zu Antonius als vielmehr, Augustus einen Strich durch die politische Rechnung zu machen, der nur darauf „bedacht“ sei, „[n]ach Rom ins Sigs-Gepräng zum Schau-Spiel uns zuführen" ${ }^{54}$ Zwar bittet sie Octavian in ihrem letzten Brief, zusammen mit Anton bestattet zu werden, doch es ist hauptsächlich ihr Stolz, als Königin, nicht als Gefangene zu sterben. Ihre Dienerinnen Eiras und Charmium folgen ihr in einer imitatio heroica nach („Hat nicht di Königin di Bahn uns schon gebrochen?“) ${ }^{55}$ und rechtfertigen in einer Periphrase des Tacitus-Mottos ihren Freitod: „Solch sterben bringet Ruhm / dis Leben Schmach und Bürde“. ${ }^{56}$ Tatsächlich empfindet Augustus den Freitod Cleopatras als Schmach. Er versucht die Tote gar zu reanimieren, bevor er ihr heroisches Sterben anerkennt und ihren ruhmvollen Tod sogar den Römern als Vorbild präsentieren will:

Welch Grimm / Cleopatra / welch wütten kam dich an?

$\mathrm{Daß}$ du so mördrisch dir / uns hast so weh gethan?

Solln itzt di Leichen uns nur unser Sigs-Fest zieren?

Last uns gleich aus Metall ihr güldnes-Bild auf-führen:

Di todten Bilder sind kein überwunden Feind /

[...]

Was wil Augustus denn di Ruhms-entseelten Glider

Auf's Schau-Gerüste stelln? Rom werffe ja darnider /

Ihr Bild aus Ertzt und Stein und glattem Helffen-Bein:

Cleopatra wird stehn / wenn Rom nicht Rom wird sein.

Vielmehr laßt uns itzt selbst ihr Bild stelln Rom für Augen /

So / wi di Schlangen ihr di edle Seel' aussaugen; ${ }^{57}$

In dieser Verabsolutierung des Heroischen spielen Präfigurate eine große Rolle. Lohensteins Protagonisten orientieren sich in Selbst- und Fremdcharakterisierungen an mythischen und historischen Vorbildern. So wird Cleopatra antonomastisch als „Zaubernde Medea“, 58 „Egyptens Helena“ ${ }^{\circ 9}$ oder „andre Zinthie“ 60 bezeichnet. Dass Lohensteins Tragödie ein Lehrstück heroischer Thanatologie ist, zeigt der Schluss: Octavian lässt sich in die Gruft der Ptolemäer führen, um das Grab Alexanders des Großen zu besuchen, den er sich wie schon Caesar zum Vorbild nimmt:

\footnotetext{
53 Ebd., V, 33-36.

54 Ebd., V, 66-67.

55 Ebd., V, 203.

56 Ebd., V, 223.

57 Ebd., V, 297-301 und 311-315.

58 Ebd., III, 289.

59 Ebd., III, 290.

60 Ebd., IV, 127.
} 
Jedoch / weil wir uns gleich itzt in der Grufft hier schau'n /

Wo Alexander ihm ließ sein Begräbnüs bau'n /

Last uns den / dem sich Glück und Tugend stets vermählet

Dem eine neue Welt zu zwingen hat gefehlet /

Den / dessen grossen Geist der Erden-Kreiß nicht schlooß

Im engen Sarche sehn. Macht Ertzt und Rügel loß.

Hier ligt der grosse Held / von dem August muß lernen:

Der Leib vergeh' in Asch / der Geist steig' an di Sternen ${ }^{61}$

Es ist einzig der Nachruhm, der über ein Leben entscheidet. Er setzt, wie Cleopatras heroischer Freitod zeigt, mindestens ein heroisches Ende voraus. Daher lehnt es Augustus ab, die Gräber der übrigen Ptolemäer anzusehen, „[m]it derer Körper Geist und Nachruhm ward begraben". 62

John Dryden: All for Love (1678)63

Im Jahre 1678 wurde John Drydens Tragödie All For Love. Or, The World Well Lost im Königlichen Theater in London uraufgeführt, erklärtermaßen „written in imitation of Shakespeare's Stile“. Tatsächlich hat Drydens heroic drama Shakespeares Antony and Cleopatra als Prätext, und seine klassizistischen Änderungen zeitigten auch einen Wandel in der Konzeption der Todesszene. Doch Dryden hat nicht nur Ort und Zeit der Handlung konzentriert sowie das Personal reduziert, sondern vor allem auf den eigentlichen Gegenspieler Octavian ganz verzichtet. Er ist als drohender Gegner immer präsent, ohne aber auf der Bühne zu erscheinen. Dies führt zu einer Interiorisierung der Konflikte und einer mitleidbasierten Sympathielenkung: Zwar geht es auch bei Dryden um Krieg und Politik, doch beide Protagonisten, Antony und Cleopatra, sind von ihrer Liebesleidenschaft füreinander so eingenommen, dass sie ihre militärischen und politischen Pflichten vernachlässigen. Auch wenn die jeweiligen Berater Cleopatra und Antony raten, ihrer Liebe zu entsagen, besetzt die Liebesleidenschaft völlig ihr Denken und Handeln. Während Antony in Melancholie versinkt, beschreibt Cleopatra ihre Leidenschaft als „transcendent passion“64 und edlen Wahnsinn: „My love’s a noble madness“.65

Drydens neue, auf Empathie setzende Ästhetik des heroic drama wirkt sich auf die Charakterisierung der Liebenden aus: Beide erscheinen als leidenschaftlich Liebende, als ,große Seelen', verbürgt durch Fremdcharakterisierungen. Wenn Antony im zweiten Akt sein Glück von Cleopatra abhängig macht („I'll not be pleased

61 Ebd., V, 393-400.

62 Ebd., V, 412.

63 Textgrundlage der folgenden Ausführungen ist: John Dryden: All For Love. Or, The World Well Lost, in: The Works of John Dryden, Bd. 13. Plays. All for Love, Oedipus, Troilus and Cressida, hg. von Edward Niles Hooker, Berkeley u. a. 1984.

64

Dryden: All For Love (Anm. 63), II, 20.

65 Ebd., II, 17. 
with less than Cleopatra" $)^{66}$ und Cleopatra ihrerseits ihn ihrer Liebe versichert („She's wholly yours. My heart's so full of joy / That I shall do some wild extravagance / Of love in public, and the foolish world, / Which knows not tenderness, will think me mad"), ${ }^{67}$ dann geschieht das jeweils in dritter Person und in erklärter Weltverachtung (,This rattle of a globe to play withal, / This gegaw world [...]").68 Die leidenschaftliche Natur beider Protagonisten verdeutlicht Dryden durch Kontrastrelationen. Zentral ist der Konfliktdialog zwischen Cleopatra, Antonys ägyptischer Mätresse, und Octavia, Antonys legitimer Ehefrau und Octavians Schwester. Im Streit der Frauen um Antony versteigt sich Cleopatra zur Behauptung: „I love him better, and deserve him more", 69 und bekennt sich zu ihrer öffentlichen Deklassierung und Selbsterniedrigung als Mätresse aus Liebe:

You bear the specious title of a wife

To gild your cause, and draw the pitying world

To favour it: the world condemns poor me.

For I have lost my honour, lost my fame,

And stained the glory of my royal house,

And all to bear the branded name of mistress.

There wants but life, and that too I would lose

For him I love. $[\ldots]^{70}$

Die Liebenden können nicht voneinander lassen: Während Antony seinerseits vergeblich versucht, Cleopatra zu entsagen und bei seiner Frau Octavia zu bleiben, erregt Cleopatra mit einer fingierten Affäre Antonys Eifersucht. Die wechselseitige Liebesleidenschaft bestimmt auch den Tragödienschluss: Als Antony die fingierte Nachricht vom Tode Cleopatras erhält, versinkt er in völlige Lethargie und fällt schließlich in sein Schwert. Cleopatras anschließende Begegnung mit dem sterbenden Antony ist viel stärker als gemeinsamer Liebestod und Post mortem-Hochzeit inszeniert. Cleopatra entspricht Antonys Bitte, ihm in den Tod zu folgen, in Form einer "short ceremony“, 71 indem sie ihn als Sieger mit dem Lorbeerkranz krönt, da nur er selbst sich besiegt habe:

[...] this laurel

Shall crown my hero's head: he fell not basely,

Nor left his shield behind him - Only thou

Couldst triumph o'er thyself, and thou alone

Wert worthy so to triumph. ${ }^{72}$

66 Ebd., II, 446.

67 Ebd., II, 447-450.

68 Ebd., II, 444-445.

69 Ebd., III, 451.

70 Ebd., III, 460-467.

71 Ebd., V, 451.

72 Ebd., V, 452-456. 
Cleopatra ihrerseits lässt sich in Erinnerung an die liebestiftende Begegnung mit Antony in Tarsos am Kydnos von ihren Dienerinnen zur ,zweiten Hochzeit ${ }^{6}$ als königliche Braut einkleiden:

[...] my second spousals

Shall match my first in glory. Haste, haste both,

And dress the bride of Antony. ${ }^{73}$

Sie setzt sich die Schlange an mit dem Wunsch: „But bring, myself, my soul to Antony"77 und bittet den Tod, sie mit dem Geliebten zu vereinen: „Oh turn me to him, / And lay me on his breast!" 75 Octavian bedenkt sie mit der zynischen Bemerkung: „Caesar, thy worst. / Now part us if thou canst“ ${ }^{\text {" }}{ }^{76}$ Anders als bei Shakespeare, wo der nüchterne Römer Octavian die Größe des toten Paares anerkennen muss, hat bei Dryden der ägyptische Isis-Priester Serapion das letzte Wort. Sein Lob, das der heroischen Liebe und dem heroischen Sterben von Antony und Cleopatra einen langen Nachruhm prophezeit, mündet in das Couplet:

[...] Sleep, blest pair,

Secure from human chance, long ages out,

While all the storms of fate fly o'er your tomb;

And fame to late posterity shall tell,

No lovers lived so great, or died so well. ${ }^{77}$

Somit ist bei Dryden der Suizid Cleopatras nur letzter Beweis ihrer heroischen Liebe, die alle Konventionen und Grenzen überschreitet. Der Unvergleichlichkeit ihrer heroischen Liebe wegen spielen Präfigurate bei Dryden so gut wie keine Rolle. Die Heroik ist in Cleopatras großer Seele und Liebesleidenschaft angelegt. Insofern stellt Dryden Cleopatras Freitod nicht als heldischen Tod, sondern als letzte Heldentat einer Heroine dar.

\section{Schluss}

Mein Überblick über Kleopatras Tod in bildkünstlerischen und literarischen Inszenierungen der Frühen Neuzeit hat gezeigt: Der Suizid der letzten ägyptischen Königin wird im Kontext des Kultes der femmes fortes medienspezifisch heroisiert, erotisiert und ästhetisiert. Konzentrieren sich die bildkünstlerischen Darstellungen auf das Moment des heldischen Todes, in dem aristokratische Würde, Mut und Schönheit zusammenkommen, integrieren die Tragödien den heroischen Moment in eine Handlung, in der die Heroik mehr oder weniger vorverlagert wird: Ist es bei Lohenstein die unbedingte Orientierung Kleopatras am Nachruhm, ist

\footnotetext{
Ebd., V, 461-463.

Ebd., V, 486-487.

Ebd., V. 498-499.

Ebd., V, 499-500.

Ebd., V, 514-518.
} 
es bei Shakespeare eine Art moralische Konversion angesichts des toten Geliebten Marcus Antonius, mit ihm zu sterben. Dagegen ist Drydens Kleopatra als große empfindsame Seele bereits eine wahre Heroine, die des Beweises eines heroischen Todes gar nicht bedarf; der Tod bestätigt ihr Heldinnentum nur noch. Somit bezeugen die bildlichen wie literarischen Sterbeszenen eine Interiorisierung des Heroischen in der Frühen Neuzeit: Losgelöst vom Militärischen büßt die Heroik ihre geschlechtliche, kulturelle und tendenziell auch ständische Bindung ein und wird zu einer spezifischen Haltung ethisiert.

\section{Abbildungsnachreise}

Abb. 1: Alessandro Turch: La Mort de Cléopâtre. akg-images, Bildnr. AKG 310566.

Abb. 2: Guido Cagnacci: Der Tod der Kleopatra, in: Elisabeth Bronfen u. a. (Hg.): Kleopatra. Die ewige Diva (Ausstellungskatalog, Bonn), München 2013, S. 309, Nr. 171.

Abb. 3: Giovanni Francesco Guerrieri: Kleopatra, in: Elisabeth Bronfen u. a. (Hg.): Kleopatra. Die ewige Diva (Ausstellungskatalog, Bonn), München 2013, S. 299, Nr. 162.

Abb. 4: Massimo Stanzione: Kleopatra, in: Elisabeth Bronfen u. a. (Hg.): Kleopatra. Die ewige Diva (Ausstellungskatalog, Bonn), München 2013, S. 303, Nr. 165.

Abb. 5: Guido Reni: Der Tod der Kleopatra, in: Elisabeth Bronfen u. a. (Hg.): Kleopatra. Die ewige Diva (Ausstellungskatalog, Bonn), München 2013, S. 306, Nr. 168.

Abb. 6: Daniel Casper von Lohenstein: Cleopatra. Trauer-Spiel, Frankfurt/ Leipzig 1724, Frontispiz. 
